\title{
Molecular docking studies of natural compounds of naringin on enzymes involved in the urea cycle pathway in hyperammonemia
}

\author{
Ramakrishnan Arumugam ${ }^{1}$, Renuka Mani², Amalan Venkatesan', \\ Senthilmurugan Sengamalai ${ }^{3}$, Vijayakumar Natesan ${ }^{1 *}$, Sung-Jin Kim ${ }^{4 *}$ \\ ${ }^{1}$ Department of Biochemistry and Biotechnology, Annamalai University, Tamilnadu, India, ${ }^{2}$ Department of Biotechnology, \\ Periyar University, Tamilnadu, India, ${ }^{3}$ Department of Zoology, Annamalai University, Tamilnadu, India, ${ }^{4}$ Department of \\ Pharmacology and Toxicology, Metabolic Diseases Research Laboratory, School of Dentistry, Kyung Hee University, Republic \\ of Korea
}

*For correspondence: Email: kimsj@khu.ac.kr, nvkbiochem@yahoo.co.in; Tel: +82-2-961-0868, +91-04144-239343

Sent for review: 18 September 2019

Revised accepted: 16 April 2020

\begin{abstract}
Purpose: To investigate the anti-hyperammonemic activity of naringin by molecular docking via in silico studies.

Methods: Urea cycle proteins were docked to the natural compound naringin as well as a standard drug, sodium benzoate. Hydrogen bonds and binding energy were obtained using Catalytic Site Atlas and Cast P Finder Software Tool.

Results: There were six urea cycle enzymes, including $N$-acetyl glutamate synthase, carbamoyl phosphate synthase $I$, ornithine transcarbamylase, argininosuccinate synthase, argininosuccinate lyase and arginase I. On evaluating protein interactions with naringin, which is dynamically connected to the urea cycle pathway with hyperammonemia, naringin showed more hydrogen bonds and also produced higher binding energy when compared to the standard drug, sodium benzoate.

Conclusion: The results of the molecular docking study show that naringin interacts with urea cycle enzymes with more hydrogen bonds and higher bonding energy than the standard drug, sodium benzoate. This supports the hypothesis that naringin can prevent experimental hyperammonemia.
\end{abstract}

Keywords: Naringin, Sodium benzoate, Hyperammonemia, Urea cycle enzymes, In silico studies

\begin{abstract}
This is an Open Access article that uses a fund-ing model which does not charge readers or their institutions for access and distributed under the terms of the Creative Commons Attribution License (http://creativecommons.org/licenses/by/4.0) and the Budapest Open Access Initiative (http://www.budapestopenaccessinitiative.org/read), which permit unrestricted use, distribution, and reproduction in any medium, provided the original work is properly credited.
\end{abstract}

Tropical Journal of Pharmaceutical Research is indexed by Science Citation Index (SciSearch), Scopus, International Pharmaceutical Abstract, Chemical Abstracts, Embase, Index Copernicus, EBSCO, African Index Medicus, JournalSeek, Journal Citation Reports/Science Edition, Directory of Open Access Journals (DOAJ), African Journal Online, Bioline International, Open-J-Gate and Pharmacy Abstracts

\section{INTRODUCTION}

Naringin is one of the natural flavonoids present in grapes and citrus fruits. The molecular formula of naringin is $\mathrm{C}_{27} \mathrm{H}_{32} \mathrm{O}_{14}$ and its molecular weight is $580.4 \mathrm{~g} / \mathrm{Mol}$. The chemical formula of naringin is 4',5,7,-trihydroxyflavonone-7-rhamnoglucoside. Naturally, it possesses a distinct bitter taste of grapefruit juice and has strong antioxidant properties [1]. Naringin is soluble in water and can be well absorbed from the gut [2]. It has been suggested that naringin has a variety of biological properties, including anti-ulcer [3], antidiabetic [4], renoprotective [5], cardioprotective [6], anti-cancer [7], anti-mutagenic [8], and antiinflammatory effects [9], as well as neuropro- 
tective activities [10]. Moreover, naringin has no significant side effects identified, as humans have been ingesting grapes and citrus fruits for a long time [11].

Ammonia is a neurotoxin in humans as well as other living organisms. Free ammonia $\left(\mathrm{NH}_{3}\right)$ is also known to be a non-proteinaceous nitrogen compound which perfuses into the cells. $\mathrm{NH}_{3}$ is converted into urea, which takes place in the liver by six urea cycle enzymes. Later, the synthesized urea is removed via urine by the kidneys [12]. The activities of carbamoyl phosphate synthase I (CPS I), N-acetyl glutamate synthase (NAGS), and ornithine transcarbamylase (OTC) occur in the hepatic mitochondria, whereas argininosuccinate synthase (ASS), argininosuccinatelyase (ASL) and arginase I (ARG) act in the hepatic cytoplasm which regulates the urea cycle pathway [13]. Due to the deficiency of urea cycle enzymes, there is a significant increase in ammonia at the blood brain barrier, which triggers hepatic encephalopathy (HE) [14]. HE exhibits a substandard cerebral edema, such as nitrosative/oxidative stress, as well as consequent dearrangements of the signal transduction pathway, neurotransmission, oscillatory networks, and synaptic plasticity in the brain as the clinical manifestations [15]. It has been suggested that hyperammonemia could lead to the hyperactivation of NMDA receptors, ultimately causing neurotoxicity [16]. Sodium benzoate has been widely used as a preservative agent for food and beverages. In 1979, USA accepted the use of sodium benzoate for medicinal purposes, but the Food and Drug Administration (FDA) stated that its use should not exceed a maximum level of $0.1 \%$. Sodium benzoate has since been used as a marker for the treatment of $\mathrm{HE}$ patients with hyperammonemia, initially in those with urea cycle enzyme deficiencies and later in patients with cirrhosis [17]. Sodium benzoate utilizes the non-urea cycle pathway for ammonia removal in the liver. Clinical medicines which contain sodium benzoate and sodium phenylacetate have been approved by the FDA for patients with urea cycle disorders and hyperammonemia $[18,19]$. Until now, molecular mechanism triggering anti-hyperammonemic activity by naringin has not been explored. Hence, this study aimed to investigate the antihyperammonemic activity of naringin by identifying the interactions between naringin and the structure of urea cycle enzymes by molecular docking using in silico studies. It has been reported that naringin regulates the urea cycle enzymes in the liver in an ammonium chloride $\left(\mathrm{NH}_{4} \mathrm{Cl}\right)$-induced hyperammonemic experimental rat model [10]. In the present study, we explored in silico properties such as interacting residues, binding energy, the number of hydrogen bonds and the bond length of urea cycle enzymes compared to the standard drug, sodium benzoate.

\section{METHODS}

\section{Preparation of ligand structures}

The naringin and sodium benzoate structures were downloaded from the PubChem database and Drug Bank (<http: //www.drugbank.ca>). The downloaded naringin structure was converted from the .sdf format to .pdb using Openbabel. This method has frequently been used in the discovery and optimization of molecular interactions with target binding energy, interacting residues, number of hydrogen bonds and bond length characterization.

\section{Preparation of protein structures}

The crystal coordinates of proteins including carbamyl phosphate synthetase I (CPS I) [PDB ID: 5DOU], ornithine transcarbomylase (OTC) [PDB ID: 1EP9], argininosuccinate synthetase (ASS) [PDB ID: 2NZ2], argininosuccinatelyase (ASL) [PDB ID: 1AOS] and arginase I (AGR) [PDB ID: 1TBJ] were downloaded from the Protein Data Bank (PDB) (http://www.pdb.org/).

\section{Catalytic site Atlas and Cast, P Finder Software tool}

The catalytic site Atlas and Cast, P Finder software tool offers a broad range of predicted active sites. The heteroatoms were removed from the PDB file and energy was minimized using GROMOS96 to stabilize the protein molecule.

\section{Molecular docking}

To determine the structure of protein ligand interaction complex in docking, studies were carried out using Auto Dock 4. Hydrogen atoms, charges were added to protein structure and grid coordinates were calculated based upon the active site and high volume surface area of the protein. Grid co-ordinates were set to generate the grid box. The docked structure was analyzed and visualized using the Molegro molecular viewer.

\section{RESULTS}

The protein molecule crystal structure was downloaded from Protein Data Bank and 
visualized by PyMol. The energy was minimized using GROMOS96.

\section{CPS I complex interacts with standard drug sodium benzoate and natural compound naringin}

Figure $1 \mathrm{~A}$ shows that the standard drug sodium benzoate docked with CPS I and the natural drug compound naringin. The level of binding energy of CPS I with the standard drug sodium benzoate was found to be $-5.98 \mathrm{KJ} /$ Mole, with three hydrogen bond interactions at SER625, VAL626 and THR627 (Table. 1). The level of binding energy of CPS I with naringin (Figure $1 \mathrm{~B}$ ) complex was found to be $-5.10 \mathrm{KJ} /$ Mole with ten hydrogen bond interactions at SER588, GLU631, THR660, GLU714, ASN716, ARG718, SER720, ARG721 and LYS772.
A

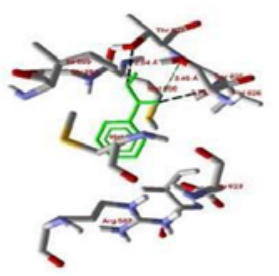

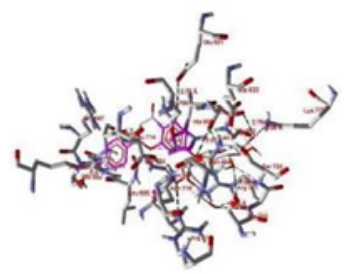

Figure 1: Molecular docking structure for CPS I with sodium benzoate and naringin. CPS I docked with the standard drug sodium benzoate (A) and natural drug compound naringin (B). The docking structures are based on hydrogen bonds and binding energy. Residues in flexible (red) and rigid (grey) modes are shown: naringin and sodium benzoate. In flexible docking, the ligands are in magenta, while in rigid docking, they are in blue

Table 1: Docking Scores of urea cycle enzymes with naringin and the standard drug sodium benzoate

\begin{tabular}{|c|c|c|c|c|c|}
\hline Protein & Drug/ligand & $\begin{array}{l}\text { Binding } \\
\text { energy } \\
(\mathrm{KJ} / \mathrm{mol})\end{array}$ & Interacting residues & $\begin{array}{c}\text { Number of } \\
\text { hydrogen } \\
\text { bonds }\end{array}$ & Bond length \\
\hline \multirow[t]{2}{*}{ CPS } & Sodium benzoate & -5.98 & $\begin{array}{l}\text { SER 625, VAL 626, } \\
\text { THR } 627\end{array}$ & 3 & $\begin{array}{l}3.45 \AA, 2.79 \AA, 2.84 \\
\AA .\end{array}$ \\
\hline & Naringin & -5.10 & $\begin{array}{l}\text { SER 588, GLU 631, } \\
\text { THR 660, GLU 714, } \\
\text { ASN 716, ARG 718, } \\
\text { SER 720, ARG 721, } \\
\text { LYS } 772 \text { (2) }\end{array}$ & 10 & $\begin{array}{l}2.41 \AA, 2.70 \AA, 2.97 \\
\AA, 2.34 \AA, 3.31 \AA, \\
3.07 \AA, 3.26 \AA, 2.98 \\
\AA .2 .75 \AA, 3.23 \AA .\end{array}$ \\
\hline \multirow[b]{2}{*}{ OTC } & Sodium benzoate & -3.24 & $\begin{array}{l}\text { SER 90, THR 91, ARG } \\
92\end{array}$ & 3 & $\begin{array}{l}3.25 \AA, 2.95 \AA, 2.91 \\
\AA\end{array}$ \\
\hline & Naringin & -6.45 & $\begin{array}{l}\text { ARG 89, THR 91, ARG } \\
\text { 92, TYR 143, ASN 198, } \\
\text { ASN 199, ASP } 263 \text { (2), } \\
\text { THR 264 }\end{array}$ & 9 & $\begin{array}{l}1.98 \AA, 3.07 \AA, 2.94 \\
\AA, 3.25 \AA, 2.94 \AA, \\
3.34 \AA, 2.68 \AA, 2.76 \\
\AA, 3.39 \AA\end{array}$ \\
\hline \multirow[b]{2}{*}{ ASS } & Sodium benzoate & -4.80 & TYR 11, ALA 36 & 2 & $2.65 \AA, 2.12 \AA$ \\
\hline & Naringin & -12.92 & $\begin{array}{l}\text { ALA 10, SER 12, GLN } \\
\text { 40, GLU 42, TYR 87, } \\
\text { GLY 117, SER 180, } \\
\text { MET 181, GLU } 183 \text { (2), } \\
\text { TYR } 282\end{array}$ & 11 & $\begin{array}{l}3.10 \AA, 3.21 \AA, \\
3.90 \AA, 2.87 \AA, 3.24 \\
\AA, 2.78 \AA, 2.97 \AA, \\
2.89 \AA, 2.89 \AA, 2.81 \\
\AA, 3.07 \AA\end{array}$ \\
\hline \multirow{2}{*}{ ASL } & Sodium benzoate & -2.41 & ILE 261 & 1 & $2.63 \AA$ \\
\hline & Naringin & -7.47 & $\begin{array}{l}\text { ILE 261, CYS 264, THR } \\
265 \text { (2), ASN 289, PRO } \\
290, \text { GLU } 294 \text { (3), ARG } \\
297 \text { (3) }\end{array}$ & 12 & $\begin{array}{l}3.80 \AA, 3.11 \AA, 3.26 \\
\AA, 3.55 \AA, 3.06 \AA, \\
3.16 \AA, \\
2.80 \AA, 3.12 \AA, 3.16 \\
\AA, 1.90 \AA, 2.67 \AA, \\
3.48 \AA .\end{array}$ \\
\hline \multirow[b]{2}{*}{ ARG I } & Sodium benzoate & -2.41 & $\begin{array}{l}\text { THR 135, THR } 136 \text { (2), } \\
\text { ASN } 139\end{array}$ & 4 & $\begin{array}{l}2.79 \AA, 3.44 \AA, 3.08 \\
\AA, 3.56 \AA\end{array}$ \\
\hline & Naringin & -3.90 & $\begin{array}{l}\text { HIS 126, ASN 130, } \\
\text { THR 135, SER 137, } \\
\text { GLY 142, ASP } 181 \text { (2), } \\
\text { ASP } 183\end{array}$ & 8 & $\begin{array}{l}3.32 \AA, 3.32 \AA, 3.36 \\
\AA, 3.11 \AA, 3.26 \AA, \\
2.73 \AA, 3.07 \AA, 2.73 \\
\AA .\end{array}$ \\
\hline
\end{tabular}


OTC complex interacts with standard drug sodium benzoate and natural compound naringin

The protein molecule OTC is docked with sodium benzoate and naringin. Figure $2 \mathrm{~A}$ shows the molecular docking structure of sodium benzoate. OTC had a binding energy of $-3.24 \mathrm{KJ} /$ Mole, with three hydrogen bond interactions at SER90, THR91 and ARG92 (Table 1). In contrast, the structural affinity of naringin with the OTC complex appeared as $-6.45 \mathrm{KJ}$ mole binding energy with nine hydrogen bond interactions at ARG89, THR91, ARG92, TYR143, ASN198, ASN199, ASP263 and THR264 (Table 1). Figure 2 B shows that the naringin complex had better structural affinity than the standard drug sodium benzoate; this might be due to its natural origin with greater binding energy and an increased number of hydrogen interactions.
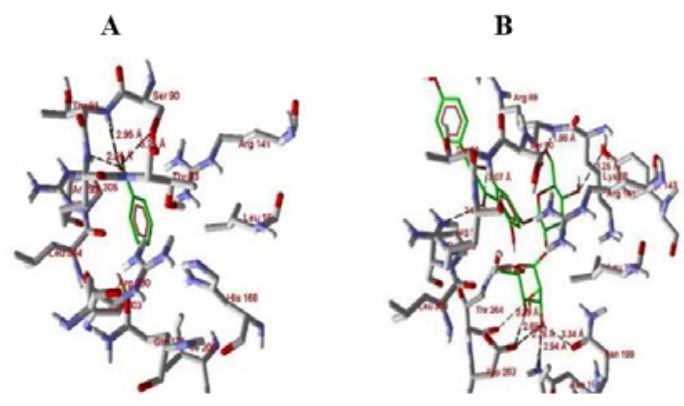

Figure 2: Molecular docking structure for OTC with sodium benzoate and naringin. Subjected to docking studies against an OTC protein molecule, naringin (B) is compared with the standard, sodium benzoate $(A)$. It shows a complex based on higher binding energy and the number of hydrogen bonds. Therefore, fluorescence quenching occurred

\section{ASS complex interacts with standard drug sodium benzoate and natural compound naringin}

When another target molecule, ASS, was docked with the standard drug sodium benzoate and the natural compound naringin, the binding energy between sodium benzoate and ASS was -4.80 $\mathrm{KJ} /$ Mole, with two hydrogen bond interactions at TYR11 and ALA36 (Figure 3 A). In contrast, the docked structure of naringin and ASS had binding energy of $-12.92 \mathrm{KJ} /$ Mole, with 11 hydrogen bond interactions identified at ALA10, SER12, GLN40, GLU42, TYR87, GLY117, SER180, MET181, GLU183 and TYR282 (Figure $3 \mathrm{~B})$. Comparatively, naringin complex had better docking scores than the sodium benzoate complex, as evidenced by its higher binding energy and more hydrogen bond interactions.

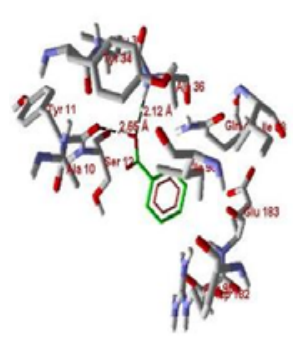

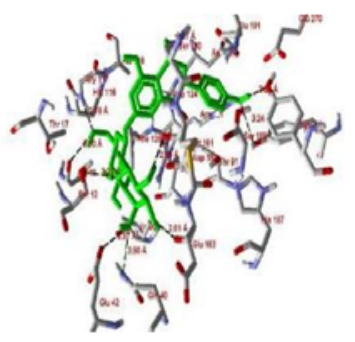

Figure 3: Molecular docking structure for ASS with sodium benzoate and naringin. Docking with ASS and sodium benzoate $(A)$ or naringin $(B)$ based on higher residues in flexible (red) and rigid (grey) modes are shown. In flexible docking, the ligands are in green, while in rigid docking, they are in blue

\section{ASL complex interacts with standard drug sodium benzoate and natural compound naringin}

Sodium benzoate and natural compound naringin were docked with the structure of ASL. The docked complex structure of the standard drug sodium benzoate with ASL had a binding energy of $-2.41 \mathrm{KJ} /$ Mole. Figure $4 \mathrm{~A}$ shows one hydrogen bond interaction at ILE261. The binding energy of the complex structure of naringin with ASL was $-7.47 \mathrm{KJ} /$ Mole, and twelve hydrogen bond interactions with residues at ILE261, CYS264, THR265 (2 bonds), ASN289, PRO290, GLU294 (3 bonds) and ARG297 (3 bonds) were identified (Figure $4 \mathrm{~B}$ ). Figure $4 \mathrm{~A}$ and $B$ comparatively show that the sodium benzoate had slightly lower binding energy, but naringin had a higher binding energy and increased range of hydrogen bond interactions than sodium benzoate.
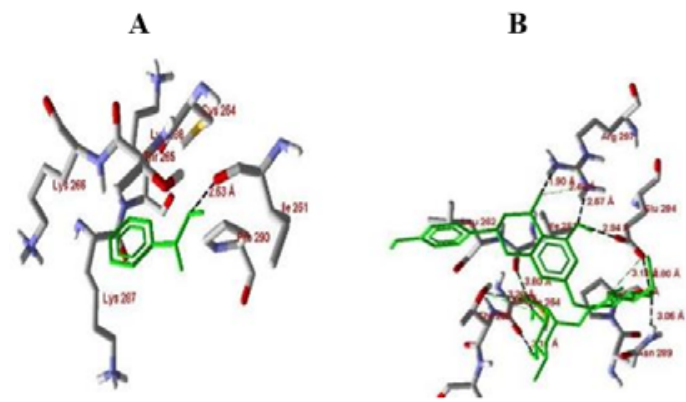

Figure 4: Molecular docking structure of ASL with sodium benzoate and naringin. Interactions between ASL and sodium benzoate (A) or naringin (B) are depicted by molecular docking studies. As well as hydrophobic interactions, some hydrogen bonding was also observed during docking with ligand-protein mixtures relevant for numerous formulations 
The ARG I complex interacts with standard drug sodium benzoate and natural compound naringin

ARG I docked with the standard drug sodium benzoate and naringin. The complex structure of AR I and the standard drug sodium benzoate had a binding energy of $-2.41 \mathrm{KJ} /$ Mole; four hydrogen bond interactions at THR135, THR136 (2 bonds) and ASN139 (Figure $5 \mathrm{~A}$ ) were identified. The naringin complex shows a binding energy range of about $-3.90 \mathrm{KJ} /$ Mole, with eight hydrogen bond interactions HIS126, ASN130, THR135, SER137, GLY142, ASP181 (2 bonds), and ASP183 (Figure $5 \mathrm{~B}$ ).
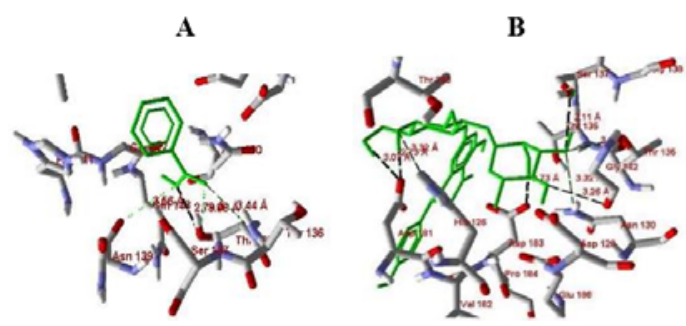

Figure 5: Molecular docking structure for ARG I with sodium benzoate and naringin. The docking poses of arginase I with sodium benzoate (A) or naringin (B) from the docking study are shown. It is clear that the drug naringin binds to where hydrophobic site residues are located. This also supports the hydrophobic character of the binding of naringin with ARG $I$, which is corroborated by the thermodynamics of the interaction. Fluorescence studies show a blue shift on complex formation, also supporting the hydrophobic association.

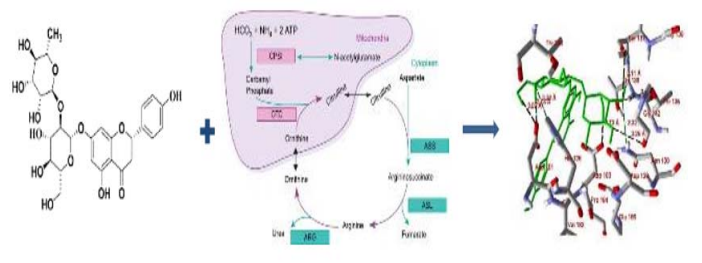

Figure 6: Graphical model of interactions between naringin with urea cycle enzymes. This model depicts the potential interaction of naringin and urea cycle enzymes

\section{DISCUSSION}

To estimate the best anti-hyperammonemic agent, the synthetic drug sodium benzoate [20] and natural compound naringin were docked with urea cycle pathway target molecules involved in the pathway to produce hyperammonemia. The drug sodium benzoate and compound naringin were docked with the protein after forming a grid; these complexes were analyzed. The best binding energy score was selected. The same structural binding predictions were followed for all of the protein and the most stable conformation was selected. A deficiency of CPS I could result in the accumulation of elevated levels of glutamine and alanine and reduced levels of citrulline and arginine in the liver tissue. The genetic location of the CPS I enzyme was chromosome $2 p \quad 35$ [21]. This study was designed for the analysis of the interaction of CPS I with naringin via molecular docking. From this result, it has been suggested that naringin may modify CPS I through binding interactions.

Increased levels of glutamine and alanine and decreased levels of citrulline and arginine were observed in OTC deficiency in liver tissues of rats. The OTC enzyme gene is localized in $X$ linked OTC (Xp21.1) [22,23]. In AL (argininosuccinic aciduria) and AS (citrullinemia) deficiencies, the level of plasma citrulline has been enhanced. If the level of serum citrulline is in the normal range, then disorders including lysinuric protein intolerance, argininemia, and the hyperammonemia-hyperornithinemia-

homocitrullinuria syndrome should be measured.

The deficiency of argininosuccinate synthase enzyme is also known as citrullinemia, which refers to higher levels of citrulline and reduced levels of arginine in the liver tissue of rats. The gene sequences responsible for this disorder are present on chromosome 9q34. Affected patients are able to partially incorporate the waste nitrogen into urea-cycle intermediates [24], making the treatment much easier. Our findings support the ASS molecular docking analysis, and indicate that naringin could be rewired back to the ASS enzyme mechanism via binding interactions.

A deficiency of argininosuccinatelyase leads to the elevation of citrulline and argininosuccinic acid and decreased levels of arginine in the liver tissue of rats. The gene for this ASL enzyme is present on chromosome AR-ASL 7cen-q11.2 $[22,23]$. Our results suggest that the structure of naringin may alter the structure of ASL through the binding interaction. ASL deficiency is also known as argininosuccinic aciduria (ASA); the diagnosis is suspected by monitoring hyperammonemia in the presence of enhanced $A L$ levels (without acidosis) in the plasma as well as the urine. Measuring the activity of $A L$ range in erythrocytes or fibroblasts could verify the diagnosis [25]. Arginase I deficiency, also called hyperargininemia, causes increased levels of arginine in the liver tissue of hyperammonemic rats. The gene for this enzyme is found on 
chromosome AR-ARG1 6q23 [22,23]. Arginase deficiency may also cause systemic hypertension due to the secondarily reduced synthesis of nitric oxide. Conversely, excess argininosuccinic acid may be culpable for the liver disease observed in some ASL-deficient patients. Our findings suggest that naringin alters ARG through the structural binding affinity [26].

\section{CONCLUSION}

Naringin can play an important role in enzyme catalysis which depends on the delicate balance of energy for different reaction pathways while participating in energy-based metabolic reactions. Thus, naringin is the most active natural compound exhibiting more energy bonds and structural interactions with urea cycle enzymes than the standard drug. Hence, it is proposed that naringin can exert a potential chemopreventive effect on urea cycle disorders as well as hyperammonemic diseases. Since hyperammonemia is an important cause of neurotoxicity via the hyperactivation of NMDA receptor (26), it is reasonable to speculate that naringin may also have a neuroprotective effect. Therefore, this study reveals that naringin is a potentially useful compound for the prevention of neurodegenerative diseases.

\section{DECLARATIONS}

\section{Acknowledgement}

This research was financially supported by DSTSERB (AST No: SB/YS/LS-286/2013), New Delhi, India. The support is greatly appreciated

\section{Conflict of interest}

No conflict of interest is associated with this work.

\section{Contribution of authors}

We declare that this work was done by the authors named in this article and all liabilities pertaining to claims relating to the content of this article will be borne by the authors.

\section{Open Access}

This is an Open Access article that uses a funding model which does not charge readers or their institutions for access and distributed under the terms of the Creative Commons Attribution License (http://creativecommons.org/licenses/by/ 4.0) and the Budapest Open Access Initiative (http://www.budapestopenaccessinitiative.org/rea d), which permit unrestricted use, distribution, and reproduction in any medium, provided the original work is properly credited.

\section{REFERENCES}

1. Jung UJ, Kim MS, Lee JS, Lee MK, Kim HO, Park EJ, Kim HK, Jeong TS, Choi MS. Naringin supplementation lowers plasma lipids and enhances erythrocyte antioxidant enzyme activities in hypercholesterolemic subjects. Clin. Nutr. 2003; 22:561-568.

2. Choudhury R, Chowrimootoo G, Srai K, Debnam E, C.A. Rice-Evans. Interactions of the flavonoid naringin in the gastrointestinal tract and the influence of glucosylation. Biophys. Res. Commun. 1999; 265:410-415.

3. Martin MJ, Marhuenda E, Perez-Guerrero C, Franco JM. Anti-ulcer effect of naringin on gastric lesions induced by ethanol in rats. J. Pharm. 1994; 49:144-150.

4. Punithavathi VR, Anuthama $R$, Prince PS. Combined treatment with naringin and vitamin $C$ ameliorates Streptozotocin-induced diabetes in male Wistar rats. J. Applied Toxicol. 2008; 28:806-813.

5. Singh $D$, Chander. V. Chopra. Protective effect of naringin, a bioflavonoid on Glycerol-induced acute renal failure in rat kidney. Toxicol. 2004; 201:143-151.

6. Rajadurai M, Prince PSM. Preventive effect of naringin on isoproterenol-induced cardiotoxicity in Wistar rats: An in vivo and in vitro study. Toxicol. 2007; 232:216-225.

7. Benavente-Garcia O, Castillo J. Update on uses and properties of citrus flavonoids: New findings in anticancer, cardiovascular and anti-inflammatory activity. J Agric Food Chem. 2008; 56:6185-6205.

8. Choi JS, Park KV, Moon SH, Rhee SH, Young HS. Antimutagenic effect of plant flavonoids in the Salmonella assay system. Pharmacal Res. 1994; 17:71-75.

9. Gonzalez-Gallego J, Sanchez-Campos S, Tunon MJ. Anti-inflammatory properties of dietary flavonoids, Nutr Hosp. 2007; 22:287-293.

10. Ramakrishnan A, Vijayakumar N. Urea cycle pathway targeted therapeutic action of naringin against ammonium chloride induced hyperammonemic rats. Biomed. Pharmacother. 2017; 94:1028-1037.

11. Choe SC, Kim HS, Jeong TS, Bok SH, Park YB. Naringin has an anti-atherogenic effect with the inhibition of intercellular adhesion molecule-I in hypercholesterolemic rabbits. J. Cardiovasc. Pharmacol. 2001; 38:947-955.

12. Brusilow SW, Maestri NE. Urea cycle disorders: Diagnosis, pathophysiology and therapy. Adv. Pediatr. 1996; 43:127-170.

13. Crombez EA. Cederbaum SD. Hyperargininemia due to liver arginase deficiency. Mol Gene Metab. 2005; 84:243-251.

14. Todd Frederick R. Current concepts in the pathophysiology and management of hepatic encephalopathy. Gastroenterol. Hepatol. 2011; 7:222233.

Trop J Pharm Res, May 2020; 19(5): 1042 
15. Häussinger $D$, Sies $H$. Hepatic encephalopathy Clinical aspects and pathogenetic concepts. Arch. Biochem. Biophys. 2013; 536:97-100.

16. Llansola $M$, Rodrigo $R$, Monfort $P$, Montoliu $C$, Kosenko E, Cauli O, Piedrafita B, El Mlili N, Felipo V. NMDA receptors in hyperammonemia and hepatic encephalopathy. Metab. Brain Dis. 2007; 22:321-335.

17. Nettis E, Colanardi M.C, Ferrannini A. Sodium benzoateinduced repeated episodes of acute urticaria/angioedema: a randomised controlled trial. Br. J. Dermatol. 2004; 151:898-902.

18. Summar ML, Dobbelaere $D$, Brusilow $S$, Lee $B$. Diagnosis, Symptoms, Frequency and Mortality of 260 Patients with Urea Cycle Disorders from a 21-Year, Multicentre Study of Acute Hyperammonaemic Episodes. Acta Paediatr. 2008; 97:1420-1425.

19. Matoori S, Leroux JP. Recent Advances in the Treatment of Hyperammonemia. Adv. Drug Deliv. Rev. 2015; 90:55-68.

20. Misel ML, Gish RG, Patton H, Mendler M. Sodium Benzoate for treatment of hepatic encephalopathy. Gastroenterol. Hepatol. 2013; 4:219-227.
21. Batshaw ML, Brusilow SW. Asymptomatic hyperammonemia in low birth weight infants. Pediatr. Res. 1978; 12:221-224.

22. Connelly A, Cross JH, Gadian DG, Hunter JV, Kirkham FJ, Leonard JV. Magnetic resonance spectroscopy shows increased brain glutamine in ornithine carbamoyl transferase deficiency. Pediatr. Res. 1993; 33:77-81.

23. Tuchman $M$, proceedings of a consensus conference for the managements of patients with urea cycle disorders. J. Pediatr. 2001; 138:1-80.

24. Summar ML. Urea Cycle Disorders Overview. In: Pagon RA, Bird TC, Dolan CR, Stephens K, eds. Gene Reviews [Internet]. Seattle (WA): University of Washington, Seattle: 1993-2003 Apr 29.

25. Braissant $O$. Current concepts in the pathogenesis of urea cycle disorders. Mol. Genet. Metab. 2010; 100(Suppl1): S3-S12.

26. Kosenkov AM, Gaidin SG, Sergeev Al, Teplov IY, Zinchenko VP. Fast Changes of NMDA and AMPA Receptor Activity Under Acute Hyperammonemia in Vitro. Neurosci. Lett. 2018; 686:80-86. 